\title{
Collapse of a transverse-mode continuum in a self-imaging photorefractively pumped ring resonator
}

\author{
Mark Saffman, Don Montgomery, and Dana Z. Anderson \\ Department of Physics and Joint Institute for Laboratory Astrophysics, University of Colorado, Boulder, Colorado $80309-0440$
}

Received November 2, 1993

\begin{abstract}
We demonstrate the collapse of a continuum of transverse modes in a self-imaging ring resonator that is photorefractively pumped. The resulting localized mode has an arbitrary transverse location. The mode collapse results from placing saturable photorefractive gain and loss media in spatially conjugate resonator planes. The transverse position of the localized mode is unstable under small cavity misalignments, and it drifts across the transverse aperture while retaining its spatial form.
\end{abstract}

The transverse-mode profile observed in laser oscillators results from the interplay of the linear boundary conditions imposed by the optical cavity and the saturating nonlinearity of the active medium. It is typically the optical cavity that exercises the largest influence on the mode structure. For example, lowpower laboratory gas lasers that are operated with a stable cavity have a low-order Hermite-Gaussian mode as the output mode. The opposite situation, in which the nonlinearity of the active medium plays the dominant role in the structure of the transverse mode, is the subject of this Letter. We demonstrate the nonlinear formation of localized modes, at arbitrary transverse locations, in a self-imaging ring resonator with saturable photorefractive gain and loss. ${ }^{1}$

The self-imaging ring resonator ${ }^{2}$ is an ideal system for studying the influence of nonlinearity on mode structure because the optical cavity plays little role in selecting the spatial mode. Any transverse optical field profile is imaged onto itself after one cavity round trip. Hence the self-imaging ring may be described alternatively as modeless or as supporting a continuum of transverse modes. In the presence of transverse aperturing the mode continuum is replaced by a discrete set of modes. However, in the experiments reported here, the resonator Fresnel number is $\mathcal{F} \sim 240$, so there are a large number of nearly equivalent transverse modes available.

Recent related research in which multimode oscillation transforms spontaneously into near singlemode oscillation has been reported in a phaseconjugating photorefractive oscillator with a chemical saturable absorber by Fischer et $a l^{3}$ and in a dye-laser system by Bazhenov et al. ${ }^{4}$ There are similarities between the experiment reported here and those of Refs. 3 and 4 . All three demonstrations of transverse-mode control rely on the presence of saturable gain and loss media at spatially distinct locations. There are also significant differences between the experiments. 'The present study uses two-beam coupling in a photorefractive crystal as a saturable loss mechanism, as opposed to a chemical saturable absorber. Furthermore, although mode formation at arbitrary transverse locations was reported in
Ref. 4, it is unclear whether the mode could form at an arbitrary transverse location in the geometry used in Ref. 3.

The resonator described here has features in common with the single-transverse-mode ring resonator with photorefractive gain and loss that was shown to exhibit bistability and self-pulsing. ${ }^{5}$ The temporal dynamics observed in the single-mode ring resonator are due to the competitive interaction of the gain and loss. In the self-imaging ring resonator the temporal dynamics are combined with transverse spatial dynamics, giving mode collapse. Juxtaposition of saturable gain and loss in conjugate resonator planes that are connected by a spatial Fourier transform leads to cooperative transverse dynamics. Both gain and loss drive the system to a localized mode of well-defined size. The subsequent dynamics of the localized mode depend strongly on the cavity alignment. Numerical simulations show that in a perfectly aligned self-imaging resonator the localized mode has a stable transverse location. A perfectly aligned system would thus be a continuous version of the winner-take-all dynamics implemented previously in a system of five coupled, but discrete, photorefractive resonators. ${ }^{6}$ However, perfect alignment is not obtainable experimentally. Experimental observations reveal that, when the optical cavity is misaligned by a small amount, such that the round-trip phase mismatch is $\sim \pi$ across the resonator aperture, the position of the localized winning mode drifts continuously across the transverse resonator aperture. ${ }^{7}$ Numerical studies of this system show that the drift motion persists for arbitrarily small cavity misalignment. When the transverse misalignment is many $\pi$, the localized modes are observed to be experimentally stable but are restricted to positions on discrete lines, corresponding to the equiphase contours of the cavity. In this case the resonator implements a winner-take-all dynamics that is continuous in one transverse dimension and discrete in the second transverse dimension.

The experimental setup is shown in Fig. 1. Planes labeled $G$ are imaged onto each other, and planes labeled $\mathcal{L}$ are imaged onto each other; $G$ and $\mathcal{L}$ 


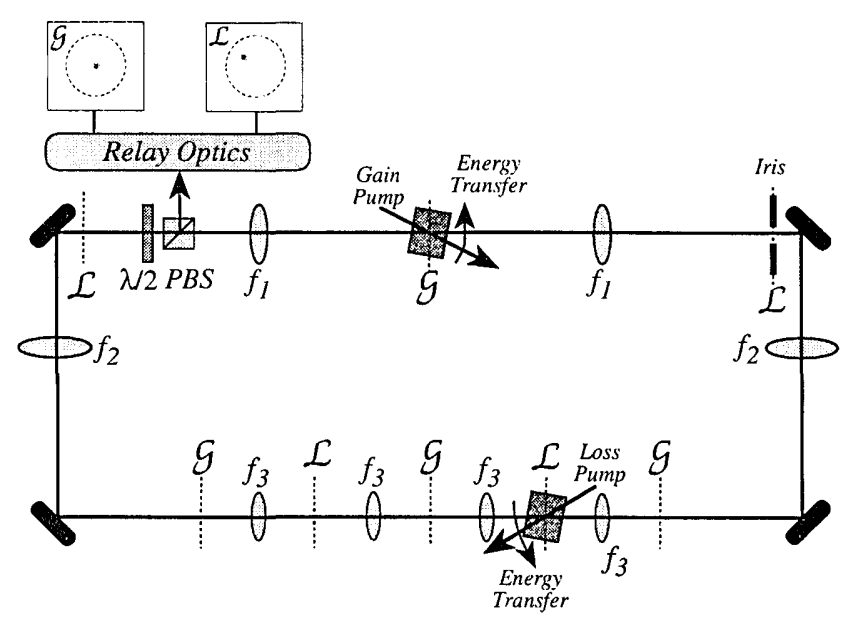

Fig. 1. Self-imaging ring resonator with photorefractive $\left(\mathrm{BaTiO}_{3}\right)$ gain and loss. The gain and loss pumps are from a cw argon-ion laser, $\lambda=514 \mathrm{~nm}$, and all beams are polarized in the plane of the figure. PBS, polarizing beam splitter. The gain pump beam has a Gaussian radius of $w_{p} \sim w_{c} \cong 130 \mu \mathrm{m}$, and the loss pump beam has a Gaussian radius of $\sim 5.5 \mathrm{~mm}$. The coupling and time constants of the gain and loss crystals $\operatorname{are}^{8} G=4.9$, $\tau_{0_{g}}=4.2 \mathrm{~s} \mathrm{~W} / \mathrm{cm}^{2}, L=2.1, \tau_{0_{l}}=0.061 \mathrm{~s} \mathrm{~W} / \mathrm{cm}^{2}$, where the small-signal gain and loss are given by $\exp (G)$ and $\exp (-L)$, respectively. The focal lengths of the lenses are $f_{1}=100 \mathrm{~mm}, f_{2}=150 \mathrm{~mm}, f_{3}=30 \mathrm{~mm}$; all lenses are confocally spaced; and the passive cavity reflectivity is $R=\exp (-C)$, where $C=3.4$.

are spatially conjugate planes. The field profiles in the planes of the gain and loss media are therefore Fourier transforms of each other. Consider first the behavior of the resonator with only gain pumping. Inside the gain medium the oscillating mode evolves to have a large spatial overlap with the pump beam such that the photorefractive gain is maximized. Letting the pump beam be a Gaussian with radius $w_{p}$ results in the oscillating mode's having an approximately Gaussian intensity distribution with radius $w_{p}$. However, there is no preferred value for the phase of the oscillating mode, and in general the variation of the phase with transverse coordinate is irregular. Because of the transverse phase variation, the Fourier transform of the field is not localized and instead fills the available aperture, as shown in Fig. 2(a). The resonator Fresnel number is given by $\mathcal{F}=r_{\text {max }}^{2} / \pi w_{c}{ }^{2}$, where $r_{\max }$ is the limiting iris radius and $w_{c}=\sqrt{\lambda f_{1} / \pi}$ is the confocal mode radius of the equivalent linear resonator. All the data presented here were taken with $r_{\max }=3.5 \mathrm{~mm}$, giving $\mathcal{F} \sim 240$. As the cavity alignment is brought closer and closer to perfect imaging, the intensity distribution observed in the loss plane exhibits large temporal fluctuations, characteristic of a metastable system.

The pump beam for the photorefractive loss crystal, which is expanded to cover the transverse aperture uniformly, is then turned on. The mode continuum collapses to a well-localized mode as shown in Fig. 2. The mode collapse dynamics do not always proceed directly to the winning transverse location. In Fig. 2(c) a location different from that of the eventual winner has the highest intensity. This type of behavior is also seen in direct numerical simulations with the oscillation seeded by noise. The time taken to collapse to a single mode depends strongly on the initial conditions. When the mode collapse proceeds directly to the winning location, the dynamical evolution may transpire as much as 20 times faster than the example shown in Fig. 2. The position of the localized mode within the transverse aperture of the resonator is arbitrary. Each time the experiment is repeated, the mode may form at a different location.

The reason for the mode collapse is that the photorefractive loss is saturable. The amount of loss decreases as the ratio of loss pump intensity to signal intensity decreases. Hence, for a given oscillating energy, the loss is minimized when the oscillating mode is well localized. The mode does not collapse to a point. Doing so would require a delocalized mode profile in the spatially conjugate plane where the gain medium is placed and, hence, low amplification. Setting the pump beam radius to $w_{p}=w_{c}$ gives a localized mode in the loss plane with radius $\left(f_{3} / f_{2}\right) w_{p}$.

In practice there is always some residual misalignment of the resonator. The misalignment leads to instability of the transverse location of the localized mode. After a spot has formed at some transverse location, either it may disappear and reappear at a different location or it may begin to execute a continuous drifting motion, as shown in Fig. 3. The details of the motion depend on the cavity alignment, the gain and loss intensities, and the coupling constants. The spot may drift to the edge of the aperture and disappear before reappearing again in its original position. Alternatively, the spot appears, moves a short distance wholly within the aperture, disappears, then reappears in the original location, and so on, in a manner that suggests a limit cycle in the dynamics. Numerical simulations indicate that the spatial instability is induced by cavity misalignment. Modeling of the misalignment as a phase wedge in two transverse dimensions reveals that the spot drifts parallel to the gradient of the wedge, with the rate of motion increasing with the severity of the misalignment. The instability persists at arbitrarily small (a) $t=0 \mathrm{~s}$
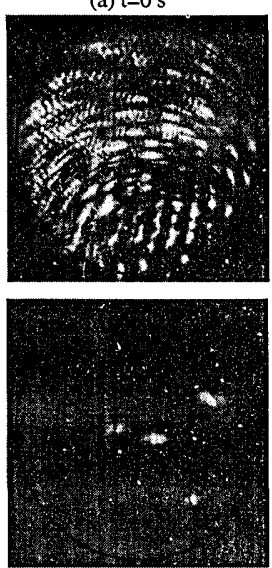

(d) $\mathrm{t}=2.0 \mathrm{~s}$ (b) $t=0.25 \mathrm{~s}$
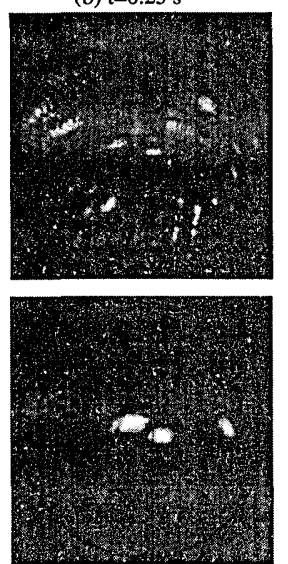

(e) $\mathrm{t}=3.0 \mathrm{~s}$ (c) $\mathrm{t}=1.0 \mathrm{~s}$
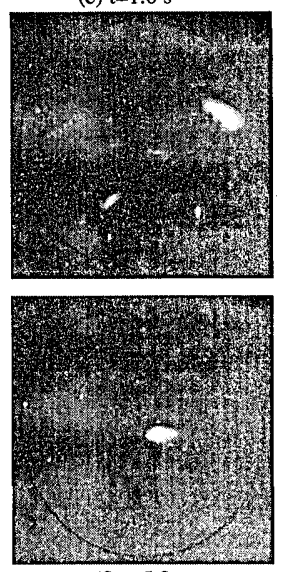

(f) $\mathrm{t}=5.0 \mathrm{~s}$
Fig. 2. Experimental observation of transverse-mode collapse. The region within the dark circle corresponds to a resonator Fresnel number of 240 . 


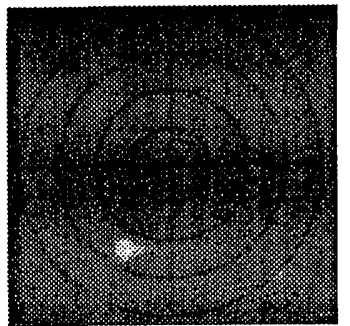

$\mathrm{t}=0 \mathrm{~s}$

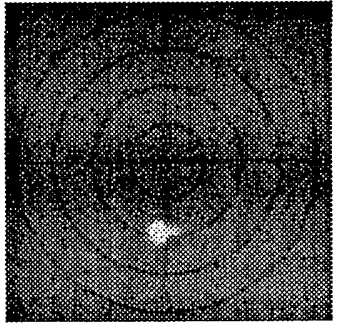

$\mathrm{t}=1 \mathrm{~s}$

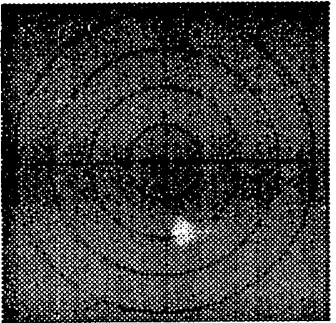

$\mathrm{t}=2 \mathrm{~s}$

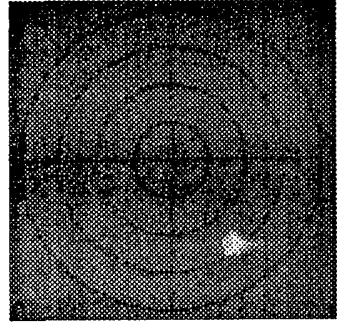

$\mathrm{t}=3 \mathrm{~s}$

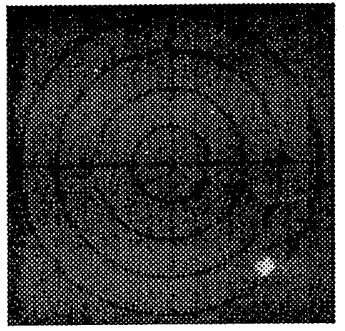

$\mathrm{t}=4 \mathrm{~s}$

Fig. 3. Experimental observation of moving localized modes. The region within the outer dark circle corresponds to a resonator Fresnel number of 240 .

misalignments, in agreement with experiment, and with arbitrarily fast loss media. This implies that spatially stable modes do not exist in a perturbed selfimaging ring resonator. The same behavior is seen numerically when the photorefractive loss is replaced by a saturable two-level system. ${ }^{9}$ This is consistent with the experimental results reported in Ref. 4.

The dynamical behavior changes character when the cavity misalignment is so large that the roundtrip phase mismatch is much greater than $\pi$. With gain only, the oscillating mode no longer fills the transverse aperture but instead appears as a fringe pattern that represents the cavity equiphase contours. When the loss pump is turned on, spatially stable localized modes form on the bright fringes. The regions that were dark fringes appear to act as barriers to the drift instability. Under these conditions the resonator behaves as a stable winner-takesall system, although the possible winning locations do not continuously fill the transverse aperture. The possible spot locations are, however, continuous along the fringes. When the cavity misalignment is made too large, localized modes will not form at all. We may derive a rough estimate for the maximum allowable number of fringes across the aperture by assuming that localized modes form only when the phase mismatch across one mode is $\leq \pi$. This gives $N \leq \sqrt{\pi \mathcal{F}} / 2 \sim 14$, where $N$ is the number of fringes.

Although we have motivated the transverse-mode collapse on the basis of steady-state gain arguments, it is necessary to account for the role of the relative time constants of the gain and loss media. Mode collapse only occurs when the loss medium is sufficiently fast relative to the gain. Recall that in the presence of a detuning $\Omega$ between the pump and signal beams the gain and loss coupling are modified by $|G| \rightarrow$ $|G| /\left(1+\Omega \tau_{g}\right)^{2}$ and $|L| \rightarrow|L| /\left(1+\Omega \tau_{l}\right)^{2}$. When the loss is much slower than the gain it is energetically favorable for the signal to avoid the loss by detuning its frequency. The intensity-dependent time constants $\left(\tau=\tau_{0} / I\right)$ with the loss pump turned on, but before the onset of mode collapse, were $\tau_{g}=0.062$ $\mathrm{s}$ (with $I_{p g}=67 \mathrm{~W} / \mathrm{cm}^{2}$ and $I_{s g}=0.60 \mathrm{~W} / \mathrm{cm}^{2}$ ) and $\tau_{l}=0.55 \mathrm{~s}$ (with $I_{p l}=0.11 \mathrm{~W} / \mathrm{cm}^{2}$ and $i_{s l}=8.6 \times 10^{-4}$ $\mathrm{W} / \mathrm{cm}^{2}$ ), where the subscripts $p$ and $s$ denote pump and signal, respectively, and $g$ and $l$ denote gain and loss media, respectively. Before mode collapse starts, the gain is approximately nine times faster than the loss. However, after the transverse mode has collapsed, the time constant of the loss medium is dominated by the signal intensity $I_{s l}=4.0 \mathrm{~W} / \mathrm{cm}^{2}$, which is much greater than $I_{p l}=0.11 \mathrm{~W} / \mathrm{cm}^{2}$. Under these conditions $\tau_{l}=0.015 \mathrm{~s} \ll \tau_{g}$. Note that the $5 \times$ demagnification provided by the combination of lenses $f_{2}$ and $f_{3}$ decreases $\tau_{l}$ by a factor of 25 . Additional experiments with the same parameters, in a resonator with no demagnifying section, and hence 25 times slower loss, did not result in transverse-mode collapse.

This research was supported by National Science Foundation grant PHY90-12244 and the Optoelectronic Computing Systems Center, a National Science Foundation Engineering Research Center. M. Saffman thanks the U.S. Air Force Office of Scientific Research for a laboratory graduate fellowship.

\section{References}

1. Preliminary accounts of this research appeared in M. Saffman, D. Montgomery, and D. Z. Anderson, in Nonlinear Dynamics in Optical Systems, Vol. 16 of 1992 OSA Technical Digest Series (Optical Society of America, Washington, D.C., 1992), paper PD3; M. Saffman, D. Montgomery, A. A. Zozulya, and D. Z. Anderson, in Digest of Conference on Photorefractive Materials, Effects, and Devices (Optical Society of America, Washington, D.C., 1993), paper ThD11.

2. J. A. Arnaud, Appl. Opt. 8, 189 (1969).

3. B. Fischer, O. Werner, M. Horowitz, and A. Lewis, Appl. Phys. Lett. 58, 2729 (1991).

4. V. Yu. Bazhenov, V. B. Taranenko, and M. V. Vasnetsov, Proc. Soc. Photo-Opt. Instrum. Eng. 1806, 14 (1993).

5. D. M. Lininger, P. J. Martin, and D. Z. Anderson, Opt. Lett. 14, 697(1989); D. M. Lininger, D. D. Crouch, P. J. Martin, and D. Z. Anderson, Opt. Commun. 76, 89 (1990).

6. C. Benkert and D. Z. Anderson, Phys. Rev. A 44, 4633 (1991).

7. A related drift-type instability in a Fabry-Perot resonator was predicted by M. Haelterman and G. Vitrant, J. Opt. Soc. Am. B 9, 1563 (1992).

8. The time constants were measured with the method described in A. Hermanns, C. Benkert, D. M. Lininger, and D. Z. Anderson, IEEE J. Quantum Electron. 28, 750 (1992).

9. A. E. Siegmam, Lasers (University Science, Mill Valley, Calif., 1986), Sec. 4.5. 